\title{
The upregulated scavenger receptor CD36 is associated with the progression of nontarget lesions after stent implantation in atherosclerotic rabbits
}

This article was published in the following Dove Press journal:

Journal of Inflammation Research

\section{Ruijian $\mathrm{Li}^{1-3, *}$ \\ Sumei Cui ${ }^{1-3, *}$ \\ Youshun $\mathrm{Xu}^{4}$ \\ Junhui $X_{\text {ing }}^{5}$ \\ Li Xue ${ }^{1-3}$ \\ Yuguo Chen ${ }^{1-3}$}

'Department of Emergency, Qilu Hospital, Shandong University, Jinan, China; ${ }^{2}$ Key Laboratory of Emergency and Critical Care Medicine of Shandong Province, Qilu Hospital, Shandong University, Jinan, China; ${ }^{3}$ Key Laboratory of Cardiovascular Remodeling \& Function Research, Chinese Ministry of Education \& Chinese Ministry of Public Health, Qilu Hospital, Shandong University, Jinan, China; ${ }^{4}$ Qilu Medical College of Shandong University, Jinan, China; ${ }^{5}$ Department of Cardiology, First Affiliated Hospital of Zhengzhou University, Zhengzhou, China

*These authors contributed equally to this work
Correspondence: Ruijian Li; Yuguo Chen Department of Emergency, Qilu Hospital, Shandong University, 107 Wenhua Xi Road, Jinan 2500I2, China Tel +8653182169325; +865318216 9319

Email liruijian2003@sdu.edu.cn; chen82169307@163.com

\begin{abstract}
Background: The incidence of recurrent cardiovascular events from the progression of nontarget lesions (NTLs) is high for percutaneous coronary intervention-treated patients. However, the underlying mechanisms have not been thoroughly elucidated.

Methods: In this study, ten atherosclerotic rabbits with multiple plaques in the upper and lower segments of abdominal aorta (group A) were randomly divided into two subgroups: group A1 underwent intravascular ultrasound examination and stent implantation in the lower segments of the abdominal aorta $(n=5)$, whereas group A2 was without stenting $(n=5)$. Group B was a control group without balloon injury. The serum levels of high-sensitivity CRP, interleukin-6 (IL-6), oxidized low-density lipoprotein, and CD36 were assessed via ELISA at five time points between the 10th and 18th weeks. The upper abdominal aorta was examined via the immunohistochemical stain and Western blotting of matrix metallopeptidase 9 (MMP-9), CD36, IL-6, and tumor necrosis factor $\alpha$.
\end{abstract}

Results: As a result, we found that stent implantation aggravated serum levels of CD36, oxidative stress, and inflammatory cytokines. Meanwhile, the upper abdominal arterial plaque burden significantly increased after stenting by intravascular ultrasound. Immunohistochemistry and Western blotting showed that the local NTLs' matrix metallopeptidase 9, CD36, IL-6, and tumor necrosis factor $\alpha$ expressions in group A1 were significantly higher than those in groups A2 and B $(P<0.05-0.01)$. More importantly, a strong correlation was identified between CD36 expression and NTLs' plaque burden before the rabbits were killed.

Conclusion: Taken together, stent implantation accelerated inflammation, induced oxidative stress, and increased the NTLs' progression, which were associated with the upregulated CD36 expression.

Keywords: inflammation, oxidative stress, CD36, atherosclerosis, stent

\section{Introduction}

Coronary heart disease (CHD) is the leading cause of morbidity and mortality worldwide, particularly in developing countries such as China. To date, percutaneous coronary intervention (PCI) with stent deployment is the most common revascularization procedure for CHD. ${ }^{1,2}$ It is reported that there are over 4,000,000 CHD patients undergoing PCI every year for the previous three decades. ${ }^{3}$ Of note, the increased use of PCI does not improve CHD patients' prognosis of recurrent cardiovascular events. It is verified that clinical events after PCI resulted from not only the in-stent restenosis but also the progression of nontarget lesions (NTLs), remote from the site 
of stent deployment. NTLs' events contributed to $46.4 \%$ of the overall events over the 5 -year follow-up. ${ }^{4}$ These results are consistent with Stone's study, ${ }^{5}$ which indicate that PCI may accelerate the progression of atherosclerosis. However, there is limited evidence from the basic research to confirm this effect and elucidate the underlying mechanisms.

Vascular injury after the cardiovascular intervention is associated with local and systemic inflammation, which depends on the type and complexity of coronary artery disease, particularly in patients with acute coronary syndromes. ${ }^{6}$ By measuring the temperature difference between the NTLs and their proximal normal vessel wall, Toutouzas et al reported that the systemic inflammatory response triggered by PCI could aggravate remote NTLs' surface heat production and local inflammatory activation. ${ }^{7}$ Apart from inflammation, oxidative stress contributes to NTLs' progression after intervention injury. Reactive oxygen species increased from 24 hours after intervention injury and lasted up to 14 days, ${ }^{6}$ thus providing the conditions for the formation of oxidized low-density lipoproteins (ox-LDL). ${ }^{8}$

CD36, a class B scavenger receptor, is a high-affinity receptor for ox-LDL, which mediates the entry of ox-LDL into the subvascular endothelium, thus leading to macrophage activation, foam cell formation, and pro-inflammatory cytokines release. ${ }^{9}$ In addition, ox-LDL via CD36 inhibits macrophage migration resulting in macrophage trapping in atherosclerotic lesions. ${ }^{10}$ Our previous clinical study investigated the function of CD36 in atherosclerosis and revealed that CD36 regulated atherosclerotic lesion progression in both the early and late stages. ${ }^{11}$ However, till now there are few studies probing the relationship between CD36 expression and NTLs' progression after vascular intervention injury.

The aim of the study was to investigate whether stentinduced inflammation and oxidative stress could affect nonintervened NTLs' progression and its relationship with CD36 expression in atherosclerotic rabbit models.

\section{Methods}

\section{Animals}

Sixteen male New Zealand rabbits $(1.5-2.5 \mathrm{~kg})$ were obtained from the Shandong Provincial Academy of Agricultural Sciences (Jinan, China) and were fed with 18 weeks of a cholesterol-rich diet ( $1 \%$ cholesterol). The animals were randomly divided into two groups: group A $(n=11)$ and group $B(n=5)$. Rabbits in group A underwent the balloon-induced abdominal aortic wall injury at the second week. Balloon-induced aortic wall injury was performed with a 4-Fr balloon catheter (balloon diameter and length $3.5 \times 15 \mathrm{~mm}^{2}$, EMERGE ${ }^{\mathrm{TM}}$ PTCA
Dilatation Catheter, Boston Scientific Corporation, Shanghai, China), which was introduced through the right femoral artery to the thoracic aorta after anesthetization with an intravenous injection of pentobarbital sodium $(30 \mathrm{mg} / \mathrm{kg})$. The balloon was inflated with saline to obtain 8 atmospheres, and the catheter was retracted down to the iliofemoral artery. This process was repeated three times in each rabbit to ensure denudation of the endothelium of the abdominal aorta and one rabbit died in this process. According to the abdominal aortic angiography at the 10th week, ten rabbits in group A with multiple plaques in the upper and lower segments of the abdominal aorta were randomly divided into two subgroups: group A1 underwent stent deployment in the lower segment according to clinical methods ( $\mathrm{n}=5$, rapamycin eluting stent, LEPU Medical, Beijing, China), whereas group A2 underwent angiography without a stent $(n=5)$. As for group $B$, the rabbits were fed a diet of $1 \%$ cholesterol, without balloon injury or stent deployment. All rabbits were sacrificed at the end of the 18 th week. NTLs were defined as the upper segment of the abdominal aorta remote from the stent at a distance of at least $5 \mathrm{~mm}$. The animal protocols were performed under the Guide for the Care and Use of Laboratory Animals and were approved by the Institutional Animal Care and Use Committee of Shandong University. ${ }^{12}$

\section{Intravascular ultrasound examination}

Intravascular ultrasound (IVUS) studies were performed using a $3.2 \mathrm{~F}$ catheter, which contained a single rotating element transducer of $40 \mathrm{MHz}$ connected to an IVUS system (iLab, Boston Scientific Corp., Fremont, CA, USA). The catheter was pulled back from the aortic arch to the abdominal aorta by a motorized withdrawal device at a constant speed of 0.5 $\mathrm{mm} / \mathrm{second}$. The following parameters were measured from the cross-sectional images: maximum diameter (Dmax), minimum diameter (Dmin), plaque area (PA), eccentricity index (EI), remodeling index (RI), the external elastic membrane area (EEMA), lumen area (LA), plaque area (PA = EEMA$\mathrm{LA})$, and plaque burden $(\mathrm{PB} \%=\mathrm{PA} / \mathrm{EEMA} \times 100 \%)$. The rabbits in groups $\mathrm{A} 1$ and $\mathrm{A} 2$ underwent IVUS at least twice (at the 10th and 18th weeks), whereas group B underwent the IVUS examination at the 18 th week prior to euthanasia.

\section{Biochemical assays}

The serum levels of total cholesterol (TC), triglyceride (TG), high-density lipoprotein cholesterol, and low-density lipoprotein cholesterol (LDL-C) were measured by enzymatic assays at the 10th, 11th, and 18th weeks. With the aim to investigate the trend over time, $4 \mathrm{~mL}$ of blood was taken from the middle ear artery at different time points (before, 1 hour, 24 hours, 7 days, 
and 8 weeks after stent). The serum levels of high-sensitivity C-reactive protein (hs-CRP), interleukin-6 (IL-6), ox-LDL, and CD36 were quantified by the ELISA. Plasma glucose concentration was also measured with the Glucose Analyzer (Yellow Spring Instrument, Yellow Springs, OH, USA).

\section{Immunohistochemistry}

At the 18th week, the rabbits were sacrificed, and the upper abdominal aorta was collected and fixed in $4 \%$ formaldehyde. Tissue samples embedded in paraffin were reacted with mouse anti-rabbit matrix metallopeptidase 9 (MMP-9) monoclonal antibody (Abcam, Cambridge, MA, USA), mouse anti-rabbit CD-36 polyclonal antibody (Bioss ANTIBODIES, Beijing, China), mouse anti-rabbit IL-6 polyclonal antibody (Bioss ANTIBODIES), and mouse anti-rabbit tumor necrosis factor $\alpha$ (TNF- $\alpha$ ) polyclonal antibody (Bioss ANTIBODIES). The sections were incubated with a goat anti-mouse peroxidaselabeled anti-body (ZSGB-BIO) as the secondary antibody at room temperature for 15 minutes. Histopathological slides were analyzed using a computer-assisted morphometric analysis system (Image-Pro Plus 5.0, Media Cybernetics, Rockville, MD, USA). The positive staining of MMP-9, CD-36, IL-6, and TNF- $\alpha$ was counted in five images of every slice under high-power fields $(\times 400)$, and three individuals independently reported the results.

\section{Western blots}

We examined the protein expression of MMP-9, CD36, IL-6, and TNF- $\alpha$ in the upper abdominal aorta by Western blot analysis. In brief, $30 \mu \mathrm{g}$ of tissue lysates were prepared as the standard protocol and separated on a $12 \%$ sodium dodecyl sulfate-polyacrylamide gel by electrophoresis. The protein bands were subsequently transferred to a polyvinylidene fluoride membrane for 1 hour and were blocked with 5\% skimmed milk for 1 hour at room temperature. The membranes were then incubated with rat anti-MMP-9 (Abcam) and rat anti-CD36/IL-6/ TNF- $\alpha$ (Bioss ANTIBODIES) at $4^{\circ} \mathrm{C}$ overnight. The membranes were subsequently incubated with human, anti-rabbit secondary antibodies (563-2; 1:1,000 dilution Zemai Biotech Corporation, Shanghai, China) for 1 hour at room temperature after three washes. Finally, the reaction was visualized using an enhanced chemiluminescence detection system (LAS MINI 4000, GE Healthcare Life Sciences, Little Chalfont, UK).

\section{Statistical analysis}

Continuous variables were reported as the mean \pm standard deviation (SD) and were analyzed using unpaired Student's t-tests and ANOVAs, whereas categorical variables were analyzed via chi-square tests. The relationship between variables was estimated by Spearman analysis. For all analyses, a $P$-value $<0.05$ was considered significant, using SPSS 17.0.

\section{Results}

\section{Stent implantation increased upper abdominal arterial plaque burden in atherosclerotic models}

Five animals per group were analyzed following our protocol (Figure 1A). Figure 1B, C shows that stent implantation effectively restored the narrowed abdominal aorta. Figure 2A shows the morphological difference of the NTLs before sacrifice by

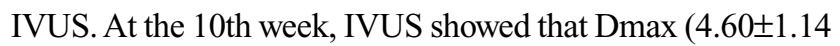
$\mathrm{mm}$ vs $3.99 \pm 0.15 \mathrm{~mm}, P<0.05)$, EEMA $\left(15.5 \pm 7.24 \mathrm{~mm}^{2}\right.$ vs $\left.8.66 \pm 0.54 \mathrm{~mm}^{2}, P<0.05\right)$, PA $\left(4.34 \pm 1.28 \mathrm{~mm}^{2}\right.$ vs $0.09 \pm 0.04$ $\left.\mathrm{mm}^{2}, P<0.05\right)$, and $\mathrm{PB}(23.57 \pm 14.63 \%$ vs $1.07 \pm 0.37 \%, P<0.05)$ were significantly increased in group A compared with those in group B (Table 1, Figure 2B). Furthermore, the above parameters were much higher in groups A1 and A2 compared with group $\mathrm{B}$ at the 18 th week (Table 1, Figure $2 \mathrm{C}-2 \mathrm{~F}$ ). Of note, an obvious increase in EI, RI, and PB was observed in group A1 compared with those in group A2 (Table 1, Figure 2E, F).

\section{Stent implantation aggravated the serum ox-LDL and glucose levels, not including TC, TG, and LDL-C}

Both Table 2 and Figure 3 show that TC, TG, and LDL-C in group A were significantly higher than those in group $\mathrm{B}$ $(P<0.01)$, while there was no significant difference between groups A1 and A2 ( $P>0.05)$. Ox-LDL level was more significantly increased in group A1 compared with group A2 $(P<0.05)$, keeping high levels till 8 weeks after stenting (Figure 4A). Also, blood glucose levels were significantly higher in group $A 1$ than those in both groups $A 2$ and $B$ at 24 hours after stenting (Figure 4B).

\section{CD36 protein level was associated with upregulated inflammation, increased oxidative stress, and abdominal arterial PB}

Immunohistochemistry showed that the local NTLs' expressions of MMP-9, IL-6, and TNF- $\alpha$ in groups A1 and A2 were significantly higher than those in group $\mathrm{B}$ (all $P<0.05$, Figure 5A, B). Furthermore, the expressions of MMP-9, IL-6, and TNF- $\alpha$ in group A1 were substantially higher than those in group A2 (all $P<0.05$, Figure $5 \mathrm{~A}-\mathrm{C}$ ). Western 


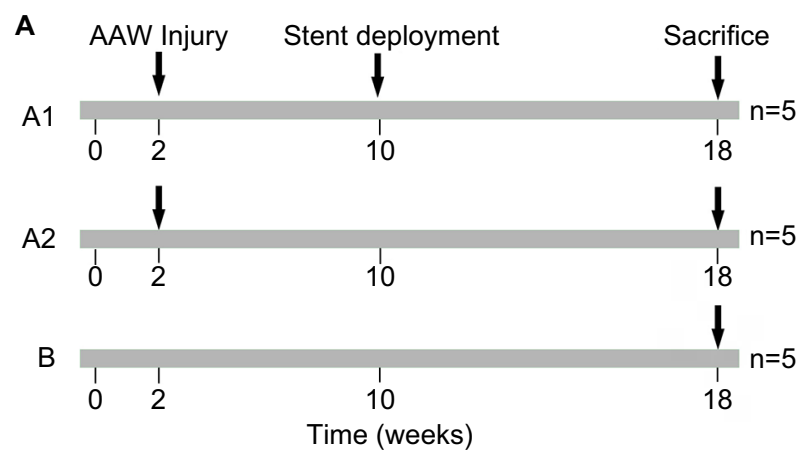

B

Before stent deployment

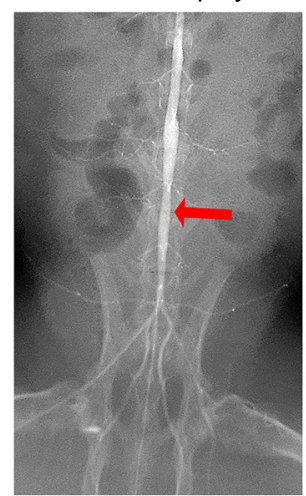

After stent deployment

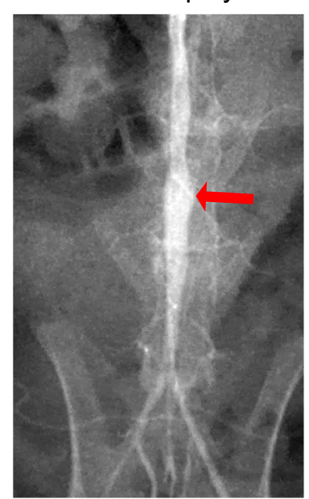

C

Before stent deployment

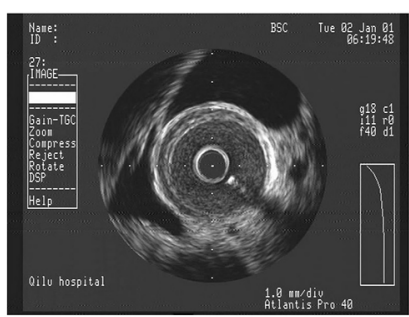

After stent deployment

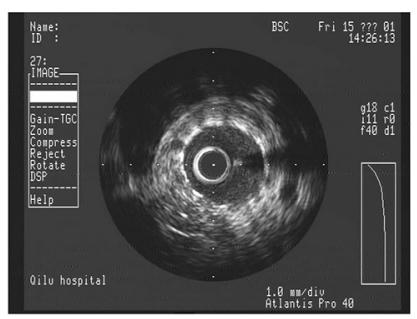

Figure I The establishment of an atherosclerotic rabbit model.

Notes: (A) Schematic showing that animals were separated into A1, A2, and B groups with different treatments. (B) Angiography showed a narrowing abdominal artery before (left) and after (right) stent deployment. Red arrows indicate the narrowing artery. (C) Intravascular ultrasonography showed that the stent was placed in the narrowing artery.

Abbreviation: AAW, abdominal aortic wall.

blotting showed similar results in which the protein expression levels of MMP-9, IL-6, and TNF- $\alpha$ in group A1 were substantially higher than the levels in groups $\mathrm{A} 2$ and $\mathrm{B}$ $(P<0.05$, Figure 5D, E).

Western blot showed CD36 levels in group A1 were significantly higher than those in group $\mathrm{A} 2$ and $\mathrm{B}(P<0.05-0.01$, Figure 6A, B). Similar results were obtained by immunostaining of CD36. The local NTLs' expressions of CD36 in group A1 were much higher than those in groups A2 and B $(P<0.05$, Figure 6C, D).

With the aim to elucidate the trend of inflammation over time, serum IL-6, hs-CRP, and CD36 were detected at five time points (before, 1 hour, 24 hours, 7 days, and 8 weeks) after stenting. IL-6 (not hs-CRP) increased at 1 hour after stenting, reached a peak at 24 hours post procedure, then declined gradually at 7 days till 8 weeks after stent (Figure 6E). However, hs-CRP increased at 7 days after stenting and continued rising until post-stent 8 weeks (Figure 6F). CD36 levels slightly increased at 1 hour after stenting, were significantly raised at 24 hours, and kept increasing until the rabbits were sacrificed (Figure 6G).
More importantly, only CD36 levels were positively correlated with elevated inflammation and oxidative stress at 24 hours after stent deployment (Figure 6H). In addition, a strong correlation was identified between CD36 expression and upper abdominal arterial PB at the 18th week (Figure 6I).

\section{Discussion}

In this study, we established an atherosclerotic model with multiple artery plaques by using the large balloon injury, which is more similar to the atherosclerotic distribution in CHD patients. The present study confirmed that stent implantation accelerated NTLs' vulnerability and progress, which can be predicted by the serum CD36 level.

IVUS, the most often used methodology to evaluate the coronary arteries in CHD patients, provided high-quality, cross-sectional imaging of the entire artery wall and important information about the build up of plaque. ${ }^{13}$ In our study, NTLs after stenting presented with greater PBs, less luminal area, and an increase in eccentric index by IVUS. Immunohistochemistry and Western blot confirmed the increased expressions of the inflammatory cytokines IL-6, TNF- $\alpha$, and 
A
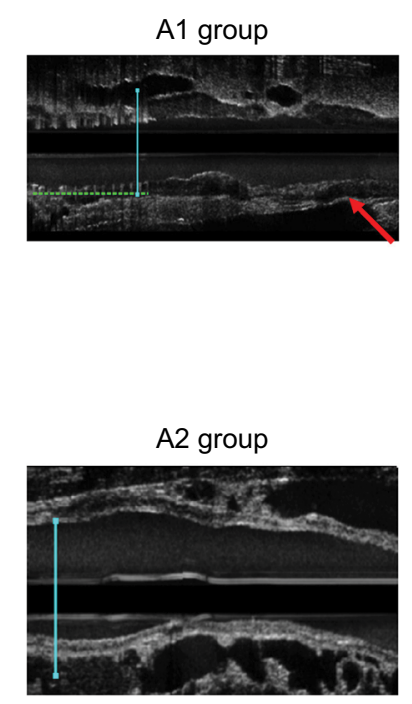

B group

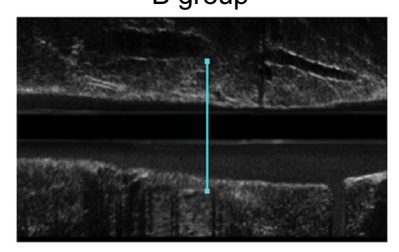

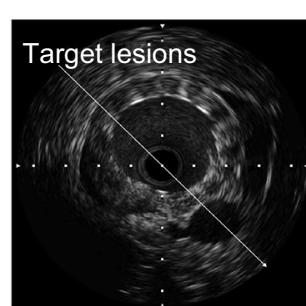

Nontarget lesions
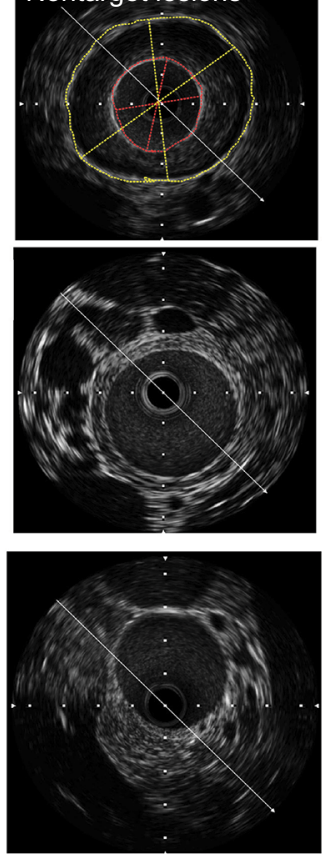

D IVUS Results at 18th week

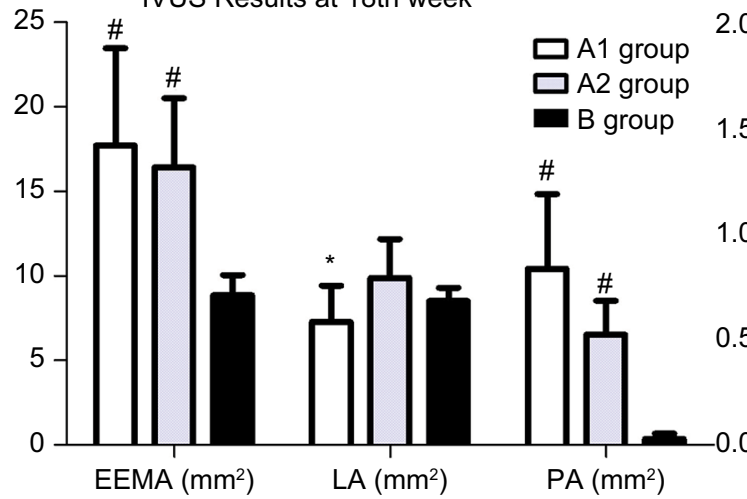

E

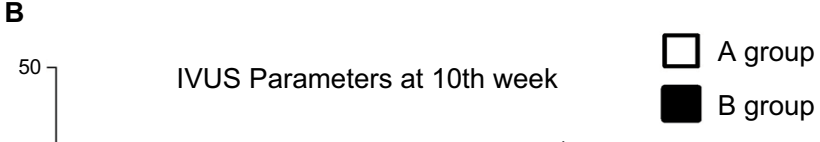

group

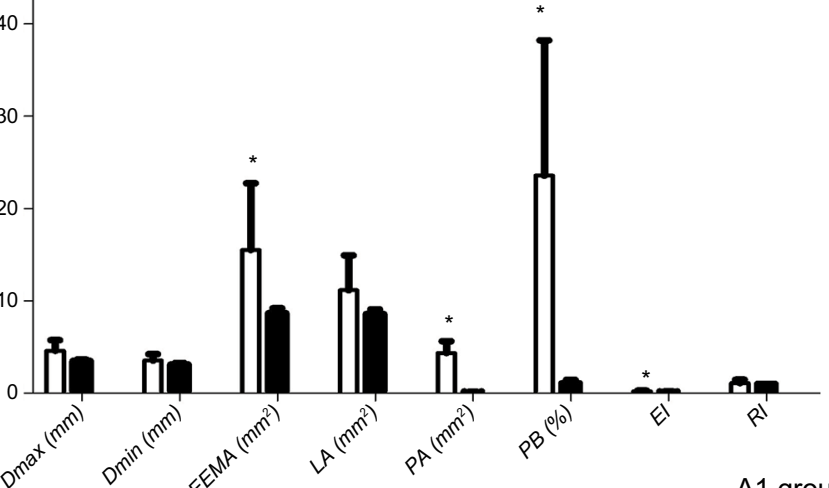

C

IVUS Results at 18th week
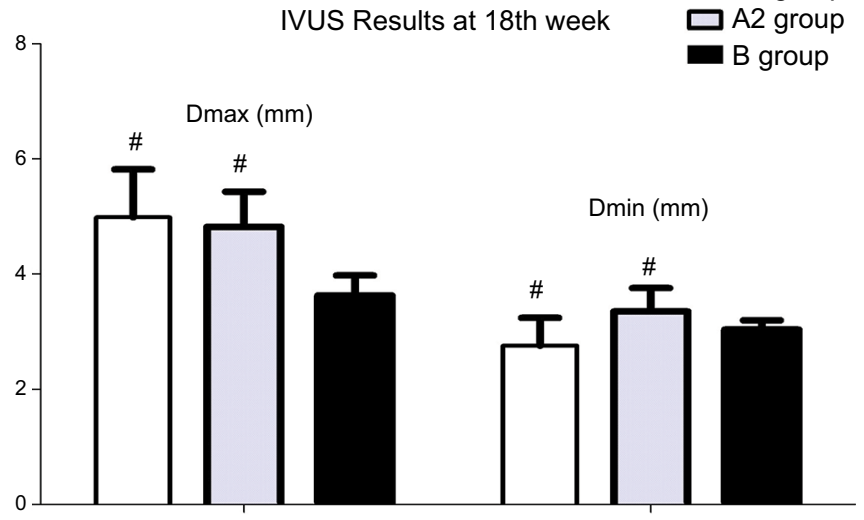

IVUS Results at 18 th week

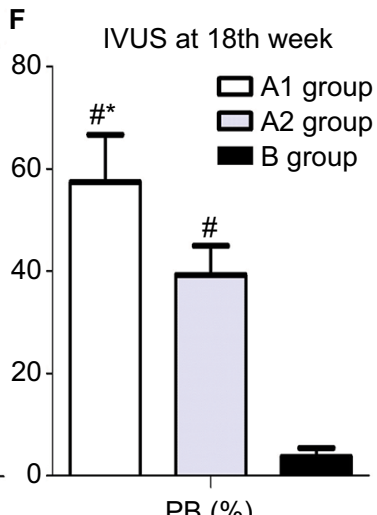

El

RI

PB (\%)

Figure 2 IVUS results at 10th week and 18th week.

Notes: (A) Representative IVUS images from three groups at the 18th week. (B) IVUS assessed the differences between groups $A$ and $B$ at I0th week. $* P<0.05$ vs group B. (C) IVUS demonstrated increased Dmax and Dmin in groups AI and A2 at the I8th week. ${ }^{P}<0.05$ vs group $B$. (D) IVUS demonstrated the change of EEMA, LA, and PA among three groups at the 18th week. ${ }^{\#} P<0.05$ vs group $B ;{ }^{*} P<0.05$ vs group $A 2$. (E) Increased $E$ I and $R I$ in groups $A I$ and $A 2$ at the I8th week by IVUS. $\#<0.05$ vs group $B$; $* P<0.05$ vs group $A 2$. (F) Increased PB in groups $A$ I and $A 2$ at the 18 th week by IVUS. ${ }^{*} P<0.05$ vs group $B$; $* P<0.05$ vs group $A 2$.

Abbreviations: Dmax, maximum diameter; Dmin, minimum diameter; EEMA, external elastic membrane area; El, eccentricity index; IVUS, intravascular ultrasound; LA, lumen area; PA, plaque area; PB, plaque burden; RI, remodeling index.

MMP-9 in local NTLs after stenting, which all potentially increased NTLs' vulnerability. These results suggested that stent implantation accelerated NTLs' vulnerability and progression by inflammation activation.
Previous studies have reported that IL-6 is an upstream inflammatory cytokine that propagates the downstream inflammatory response responsible for the progression of atherosclerosis. ${ }^{14,15}$ In this study, we determined that only 
Table I Values of IVUS for nontarget lesions

\begin{tabular}{|c|c|c|c|c|c|c|c|c|c|}
\hline Group & $\begin{array}{l}\text { Time } \\
\text { (week) }\end{array}$ & $\begin{array}{l}\text { Dmax } \\
(\mathrm{mm})\end{array}$ & $\begin{array}{l}\text { Dmin } \\
(\mathrm{mm})\end{array}$ & $\begin{array}{l}\text { EEMA } \\
\left(\mathrm{mm}^{2}\right)\end{array}$ & LA $\left(\mathrm{mm}^{2}\right)$ & PA $\left(\mathrm{mm}^{2}\right)$ & PB (\%) & EI & $\mathbf{R I}$ \\
\hline A & 10th & $4.60 \pm 1.14^{\#}$ & $3.53 \pm 0.69$ & $15.50 \pm 7.24^{\#}$ & $11.16 \pm 3.77$ & $4.34 \pm 1.28^{\#}$ & $23.57 \pm 14.63^{\#}$ & $0.22 \pm 0.07^{\#}$ & $1.09 \pm 0.36$ \\
\hline $\mathrm{Al}$ & 18th & $4.99 \pm 0.83^{\#}$ & $2.76 \pm 0.48^{\Delta \#}$ & $17.72 \pm 5.74^{\#}$ & $7.29 \pm 2.14^{* \Delta}$ & $10.42 \pm 4.43^{* \Delta \#}$ & $57.42 \pm 9.29 * \Delta \#$ & $0.54 \pm 0.07^{* \Delta \#}$ & $1.14 \pm 0.29 * \Delta \#$ \\
\hline $\mathrm{A} 2$ & 18th & $4.82 \pm 0.6 I^{\#}$ & $3.35 \pm 0.4 I^{*}$ & $16.43 \pm 4.08^{\#}$ & $9.89 \pm 2.28$ & $6.54 \pm 1.99^{\#}$ & $39.30 \pm 5.67^{\#}$ & $0.30 \pm 0.04^{\#}$ & $1.07 \pm 0.23$ \\
\hline \multirow[t]{2}{*}{ B } & 10th & $3.49 \pm 0.15$ & $3.10 \pm 0.18$ & $8.66 \pm 0.54$ & $8.57 \pm 0.5 \mathrm{I}$ & $0.09 \pm 0.04$ & $1.07 \pm 0.37$ & $0.11 \pm 0.07$ & $0.98 \pm 0.02$ \\
\hline & 18th & $3.63 \pm 0.35$ & $3.04 \pm 0.16$ & $8.87 \pm 1.19$ & $8.5 \mathrm{I} \pm 0.79$ & $0.36 \pm 0.39$ & $3.73 \pm 1.73$ & $0.16 \pm 0.05$ & $1.00 \pm 0.10$ \\
\hline
\end{tabular}

Notes: Values are the mean $\pm S D$. ${ }^{* P}<0.05$ compared with group $A 2$ at the same time; ${ }^{\triangle P}<0.05$ compared with group $A$ at 10 th week; ${ }^{\# P<0.05}$ compared with group $B$ at the same time.

Abbreviations: Dmax, maximum diameter; Dmin, minimum diameter; EEMA, external elastic membrane area; El, eccentricity index; IVUS, intravascular ultrasound; LA, lumen area; PA, plaque area; PB, plaque burden; RI, remodeling index.

Table 2 Changes in serum cholesterol $(\mathrm{mmol} / \mathrm{L})$ and triglycerides $(\mathrm{mmol} / \mathrm{L})$ in rabbits over time

\begin{tabular}{|l|l|l|l|l|l|}
\hline Group & Time (week) & TC & TG & HDL-C & LDL-C \\
\hline AI & I0th & $39.32 \pm 6.32^{*}$ & $2.46 \pm 1.39 * *$ & $3.00 \pm 0.74 *$ & $10.52 \pm 4.04^{*}$ \\
& IIth & $36.41 \pm 4.90^{*}$ & $2.22 \pm 1.06^{* *}$ & $2.97 \pm 0.91$ & $10.08 \pm 3.60^{*}$ \\
A2 & I8th & $36.26 \pm 3.0 I^{*}$ & $2.38 \pm 1.70^{* *}$ & $2.94 \pm 0.47$ & $9.66 \pm 1.8 I^{*}$ \\
& IOth & $38.06 \pm I I .14^{*}$ & $2.98 \pm 1.58^{* *}$ & $3.11 \pm 0.53^{*}$ & $12.05 \pm 4.12^{*}$ \\
& IIth & $36.16 \pm 8.28^{*}$ & $2.58 \pm 1.25^{* *}$ & $3.02 \pm 0.38$ & $11.03 \pm 2.87^{*}$ \\
B & I8th & $38.61 \pm 9.43^{*}$ & $2.84 \pm 1.18^{* *}$ & $3.07 \pm 0.27$ & $11.89 \pm 3.76 *$ \\
& IOth & $21.90 \pm 4.15$ & $0.52 \pm 0.05$ & $1.70 \pm 0.57$ & $4.49 \pm 0.35$ \\
& IIth & $22.79 \pm 3.90$ & $0.67 \pm 0.10$ & $2.08 \pm 0.30$ & $4.49 \pm 0.37$ \\
& I8th & $23.73 \pm 2.47$ & $0.65 \pm 0.05$ & $2.36 \pm 0.32$ & $5.71 \pm 1.04$ \\
\hline
\end{tabular}

Notes: Values are the mean $\pm S D$. $* P<0.05 ; * * P<0.01$ compared with group $B$ at the same time.

Abbreviations: HDL-C, high-density lipoprotein cholesterol; LDL-C, low-density lipoprotein cholesterol; TC, total cholesterol; TG, triglyceride.

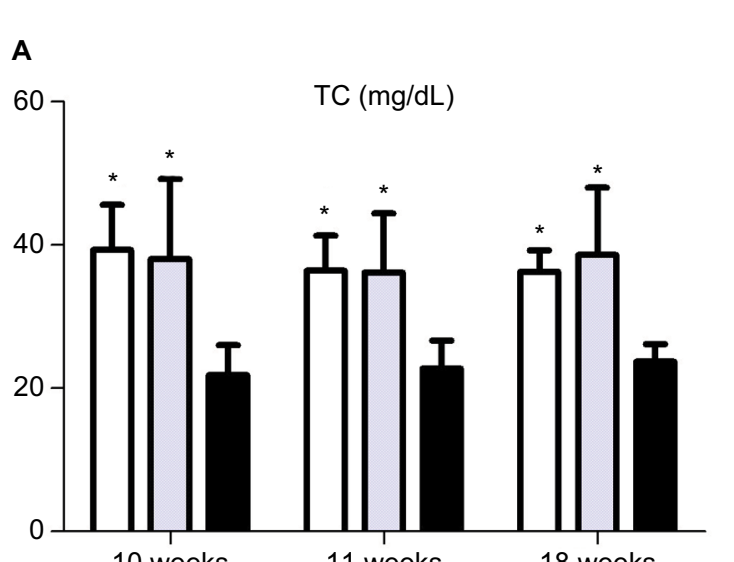

C

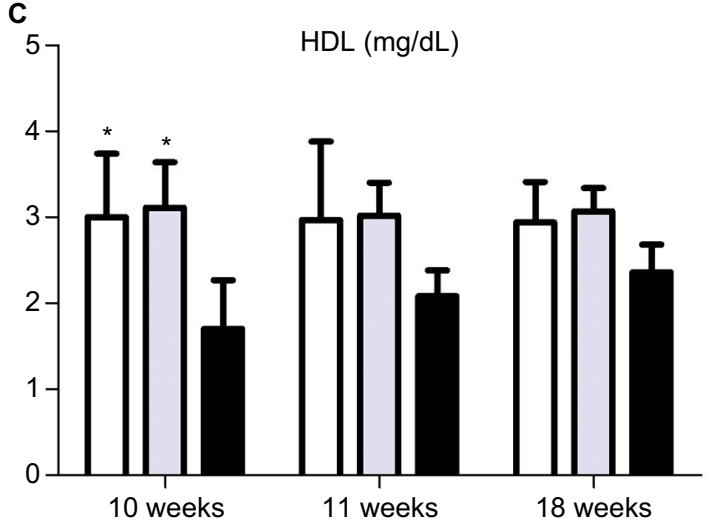

B
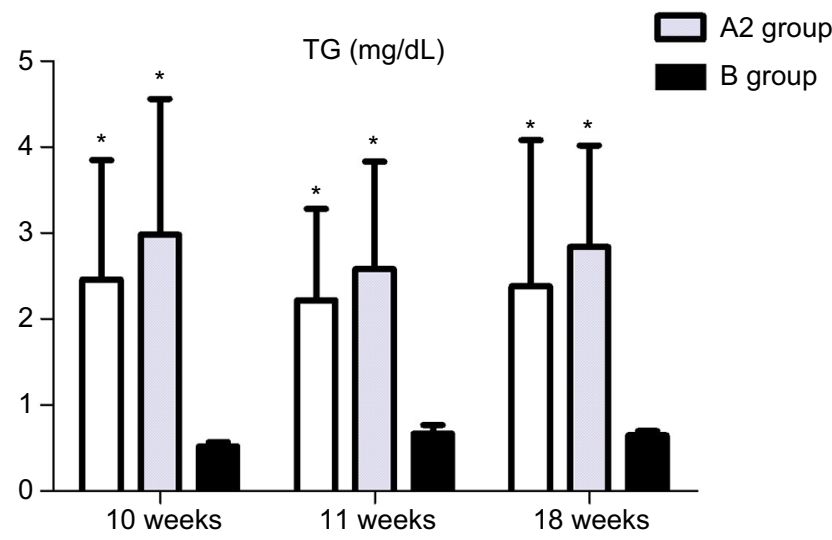

D

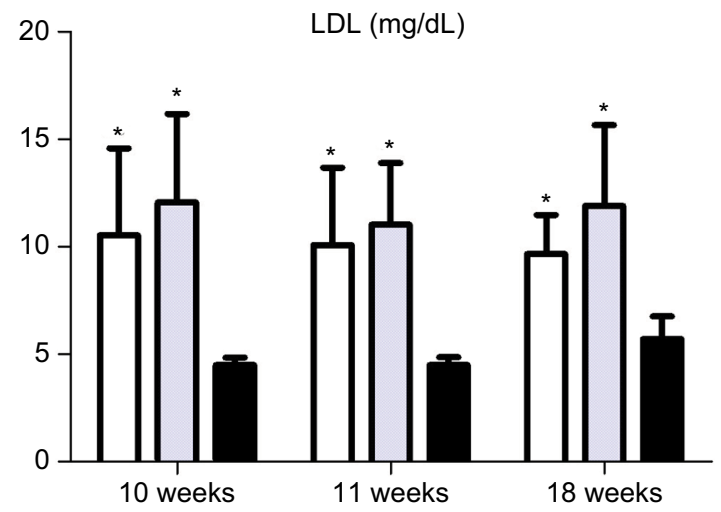

Figure 3 ELISA showed upregulated TC (A), TG (B), HDL-C (C), and LDL-C (D) in the blood at the I0th, IIth, and I8th weeks. "P<0.05 vs group B. Abbreviations: HDL-C, high-density lipoprotein cholesterol; LDL-C, low-density lipoprotein cholesterol; TC, total cholesterol; TG, triglyceride. 

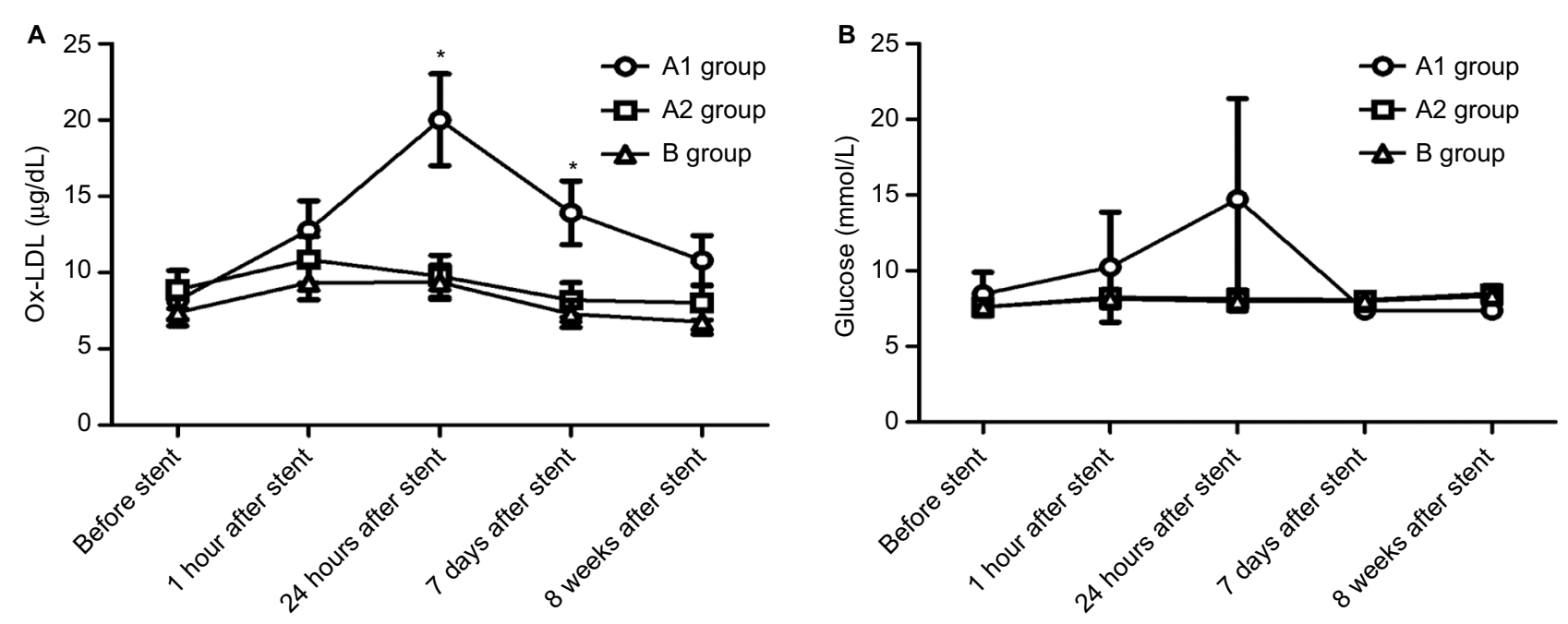

Figure 4 Stent implantation aggravated the serum ox-LDL and glucose levels.

Notes: (A) ELISA showed the blood levels of ox-LDL at five time points; ${ }^{*}<0.05$ vs ox-LDL levels before stent. (B) Glucose Analyzer was utilized to measure the plasma glucose over time.

Abbreviation: ox-LDL, oxidized low-density lipoprotein.

IL-6, not hs-CRP, significantly increased at 1 hour of rabbit abdominal arterial stent implantation. This level peaked at 24 hours and then gradually declined after 7 days for 4 weeks; however, it remained higher than the control and ballooninjury groups. Interestingly, the IL-6 levels at 24 hours post stent deployment were related to the CD36 levels. We confirmed that IL- 6 is the earliest released inflammatory factor induced by stent implantation, which was associated with CD36 upregulation. With respect to another inflammation indicator hs-CRP, we surprisingly found that the hs-CRP levels were unchanged within the first 24 hours and began to increase on the seventh day compared with the baseline levels. This trend was inconsistent with previous clinical observations. ${ }^{16,17}$ One reason for the early upregulation of hs-CRP in CHD patients after PCI may be the perioperative myocardial infarction induced by microthrombosis, ${ }^{18}$ which rarely occurred in our study because the stent site-abdominal aorta does not locate in terminal vessels. However, elevated levels of hs-CRP from the seventh day to the 18th week suggested that hs-CRP participated in the late progression of vulnerable plaques.

In addition to inflammation, ox-LDL (a marker of lipoprotein-associated oxidative stress) and blood glucose significantly increased after stenting in our study. A positive relationship between the ox-LDL, hyperglycemia, and CD36 levels was observed, suggesting that oxidative stress (represented by ox-LDL and hyperglycemia) played a central role in the initiation of $\mathrm{CD} 36 .{ }^{19-21} \mathrm{CD} 36$ belongs to class $\mathrm{B}$ scavenger receptors, which are membrane glycoproteins with a mass of $88 \mathrm{kDa}$, composed of a single protein chain. CD36 locates on the multiple cell surface, such as adipocytes, monocytes, platelets, and macrophages. The macrophage is a key in atherosclerosis progression, which takes up ox-LDL mainly through scavenger receptors. ${ }^{9} \mathrm{CD} 36$ is the most important scavenger receptor in macrophage recruitment to atherosclerotic lesions and leads to vascular wall inflammation through the combination of ox-LDL in a receptor-type manner. ${ }^{22}$ Recent studies have reported an additional atherogenic effect by platelet CD36-bound ox-LDL. Platelets interacting with ox-LDL release chemokines and mediate platelet-monocyte aggregate formation, which enhances monocytes, phenotypic changes and foam cell formation. ${ }^{23,24}$

Our previous study showed that CD36 regulated atherosclerotic lesion progression in both the early and late stages. ${ }^{11}$ Harb et al showed that the recruitment of radiolabeled macrophages to atherosclerotic lesions was reduced by pretreatment with the CD36-binding peptide EP80317. In contrast, in CD36-deficient mice, the pretreatment had no effects. ${ }^{25}$ In humans, autopsy studies performed in CHD patients have demonstrated increased expression of the CD36 receptor on macrophages, comprised within atherosclerotic plaques. Taken together, CD36 is an important receptor in atherosclerosis progression. ${ }^{26}$

In this study, we determined that the local expression of CD36 in NTLs was substantially higher after stenting than the control and vehicle groups. Moreover, the serum CD36 


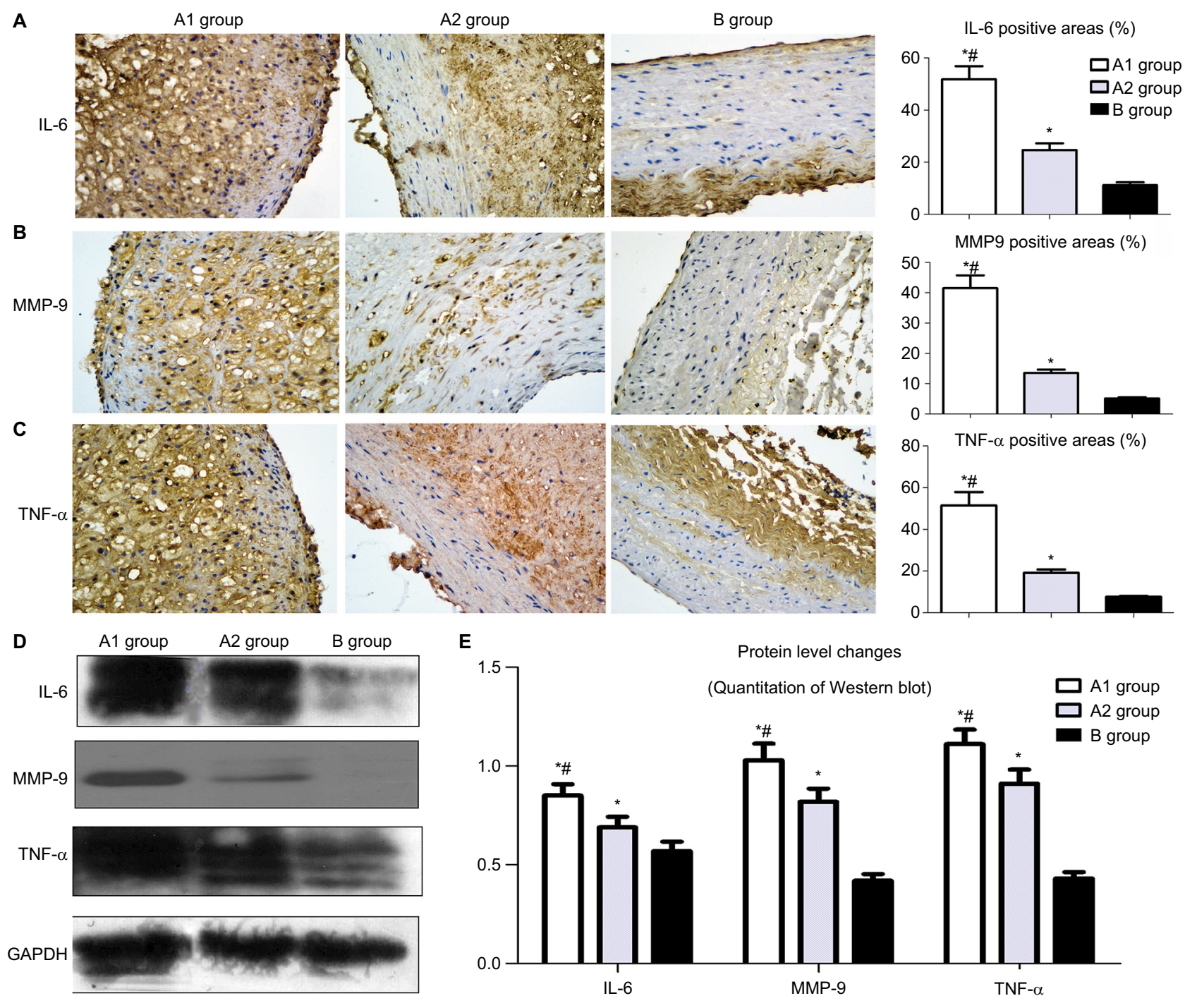

Figure 5 Effect of stent implantation on the nontarget lesions' inflammation determined by immunostaining and Western blot.

Notes: (A) Representative immunostaining images of IL-6 are shown in the left panel and quantification was achieved by normalizing IL-6 positive areas to the plaque sizes. (B) Representative immunostaining images of MMP-9 in the left panel and quantification was achieved by normalizing MMP-9 positive areas to the plaque sizes. (C) Representative immunostaining images of TNF- $\alpha$ and analysis data. (D) Western blot was used to measure the protein level changes of IL-6, MMP-9, and TNF- $\alpha$ in the upper segment of abdominal aorta. (E) Image J was used to quantify the results in Figure 5D. ${ }^{*} P<0.05$ vs group B; ${ }^{*} P<0.05$ vs group $A 2$.

Abbreviations: IL-6, interleukin-6; MMP-9, matrix metallopeptidase 9; TNF- $\alpha$, tumor necrosis factor $\alpha$.

was the only cytokine that significantly increased from the early time and maintained high levels until 8 weeks. In addition, CD36 levels in the blood were positively correlated with elevated inflammation and oxidative stress which are confirmed to accelerate the progression of NTLs in our study. More importantly, the relationship analysis showed that only CD36 levels were significantly related to the progression of PB of NTLs at the 18 th week. This observation suggests that $\mathrm{CD} 36$ has a great potential to be a novel biomarker to predict the progression of NTLs. Meanwhile, CD36 has high expression levels in the smooth muscle cells by immunostaining. But, whether the circulating CD36 originates from smooth muscle cells or not remains to be further studied. Taken together, CD36 was confirmed as a key receptor in the progression of NTLs, which might have potential implications for clinical practice.

An important limitation was that our study failed to block or interfere with CD36 expression by gene knockout or siRNA. Furthermore, atherosclerotic rabbit abdominal aortic stents were deployed without drug treatment. Moreover, animal models established by balloon injury and high-fat diet do not completely resemble atherosclerotic plaques in humans.

In this study, we confirmed that nonintervened NTLs were accelerated by stenting-induced inflammation and 

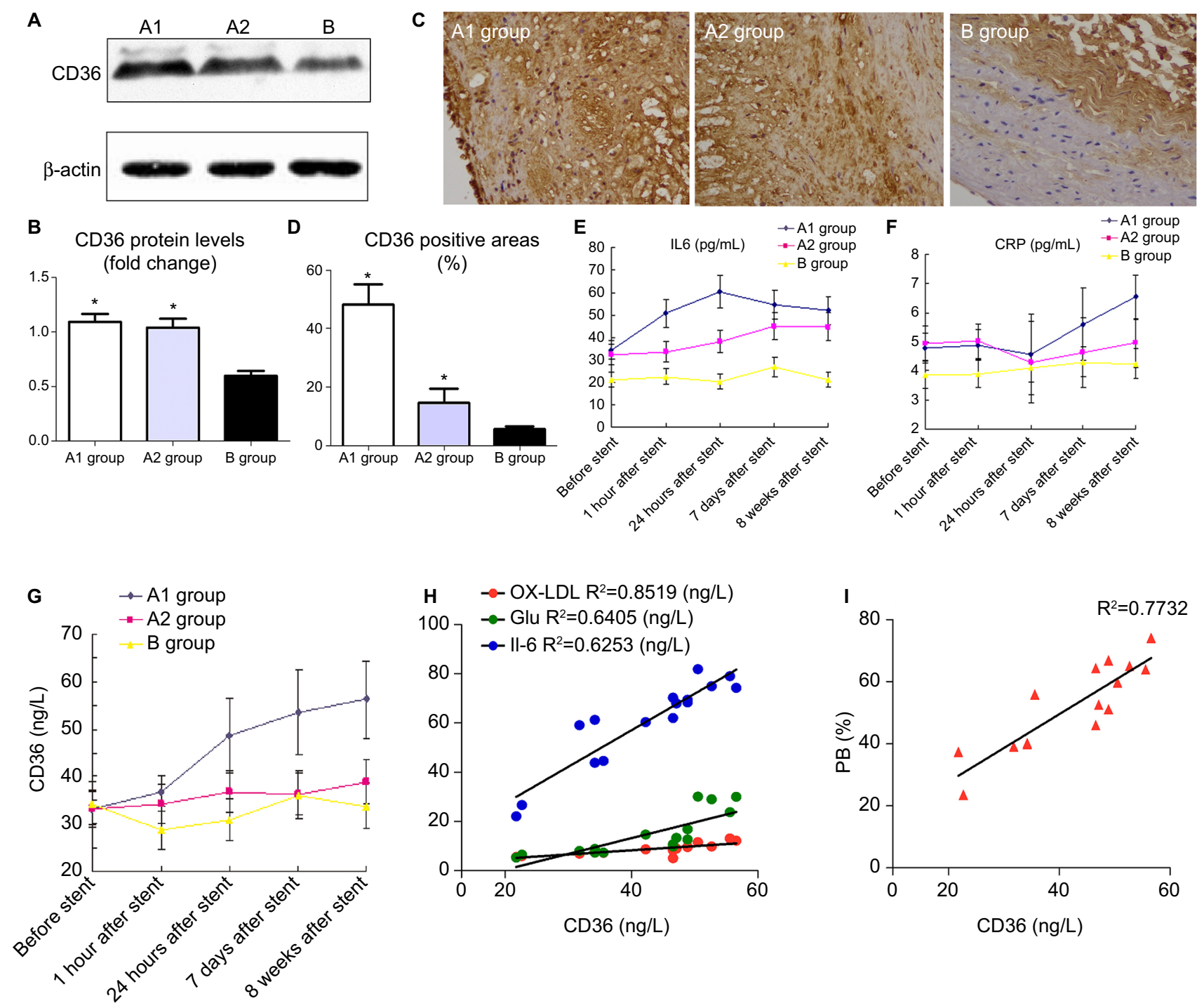

Figure 6 CD36 protein expression of the upper segment of abdominal aorta among the three groups by Western blot $(\mathbf{A})$ and quantitative analysis $(\mathbf{B}) .{ }^{*} P<0.05$ vs group $B$. (C) Representative immunostaining images of CD36 expression in the upper segment of abdominal aorta. (D) Quantitative analysis of CD36 in Figure $5 C$. "P $<0.05$ vs group B. ELISA was performed to assess the serum IL-6 protein levels (E), hs-CRP protein levels (F), CD36 protein levels (G) at five time points. (H) CD36 levels in blood were associated with ox-LDL, glucose, and IL-6 at 24 hours after stenting. (I) CD36 levels in blood were associated with PB by IVUS at I8th week.

Abbreviations: hs-CRP, high-sensitivity C-reactive protein; IL-6, interleukin-6; IVUS, intravascular ultrasound; ox-LDL, oxidized low-density lipoprotein; PB, plaque burden.

oxidative stress, which were associated with upregulated CD36 expression.

\section{Acknowledgments}

This research was supported by China Postdoctoral Science Foundation (ZR2015HM039), Shandong Province Natural Science Foundation (2015M572054), and Shandong Province Medical Science and Technology Development Plan (2013WS0216).

\section{Disclosure}

The authors report no conflicts of interest in this work.

\section{References}

1. Park HW, Yoon CH, Kang SH, et al. Early- and late-term clinical outcome and their predictors in patients with ST-segment elevation myocardial infarction and non-ST-segment elevation myocardial infarction. Int J Cardiol. 2013;169(4):254-261.

2. Deng F, Lv JH, Wang HL, Gao JM, Zhou ZL. Expanding public health in China: an empirical analysis of healthcare inputs and outputs. Public Health. 2017;142:73-84.

3. Feng C, Ji T, Liu Y, et al. Role of depression in secondary prevention of Chinese coronary heart disease patients receiving percutaneous coronary intervention. PLoS One. 2017;12(12):e0187016.

4. Boden WE, O'Rourke RA, Teo KK, et al. Optimal medical therapy with or without PCI for stable coronary disease. $N$ Engl $J$ Med. 2007;356:1503-1516.

5. Stone GW, Maehara A, Lansky AJ, et al. A prospective natural-history study of coronary atherosclerosis. N Engl J Med. 2011;364(3):226-235. 
6. Rosenbaum MA, Miyazaki K, Graham LM. Hypercholesterolemia and oxidative stress inhibit endothelial cell healing after arterial injury. $J$ Vasc Surg. 2012;55(2):489-496.

7. Toutouzas K, Drakopoulou M, Markou V, et al. Correlation of systemic inflammation with local inflammatory activity in non-culprit lesions: beneficial effect of statins. Int J Cardiol. 2007;119(3):368-373.

8. Shi Y, Niculescu R, Wang D, Patel S, Davenpeck KL, Zalewski A. Increased $\mathrm{NAD}(\mathrm{P}) \mathrm{H}$ oxidase and reactive oxygen species in coronary arteries after balloon injury. Arterioscler Thromb Vasc Biol. 2001;21(5):739-745.

9. Yamashita S, Hirano K, Kuwasako T, et al. Physiological and pathological roles of a multi-ligand receptor CD36 in atherogenesis; insights from CD36-deficient patients. Mol Cell Biochem. 2007;299(1-2):19-22.

10. Park YM, Drazba JA, Vasanji A, EgelhoffT, Febbraio M, Silverstein RL. Oxidized LDL/CD36 interaction induces loss of cell polarity and inhibits macrophage locomotion. Mol Biol Cell. 2012;23(16):3057-3068.

11. Li RJ, Yang M, Li JF, Xue L, Chen YG, Chen WQ. Circulating CD36 and fractalkine levels are associated with vulnerable plaque progression in patients with unstable angina pectoris. Clin Exp Pharmacol Physiol. 2014;41(11):863-869.

12. Committee for the Update of the Guide for the Care and Use of Laboratory Animals, Institute for Laboratory Animal Research, Division on Earth and Life Studies, National Research Council of the National Academies. Guide for the Care and Use of Laboratory Animals. 8th ed. Washington: National Academies Press; 2011.

13. Mehran R, Mintz GS, Hong MK, et al. Validation of the in vivo intravascular ultrasound measurement of in-stent neointimal hyperplasia volumes. J Am Coll Cardiol. 1998;32:794-799.

14. Aggarwal A, Schneider DJ, Terrien EF, Gilbert KE, Dauerman HL. Increase in interleukin-6 in the first hour after coronary stenting: an early marker of the inflammatory response. J Thromb Thrombolysis. 2003;15(1):25-31.

15. Segev A, Kassam S, Buller CE, et al. Pre-procedural plasma levels of C-reactive protein and interleukin-6 do not predict late coronary angiographic restenosis after elective stenting. Eur Heart J. 2004;25(12):1029-1035.
16. Szmitko PE, Wang CH, Weisel RD, de Almeida JR, Anderson TJ, Verma S. New markers of inflammation and endothelial cell activation: Part I. Circulation. 2003;108(16):1917-1923.

17. $\mathrm{Xu} \mathrm{YL,} \mathrm{Li} \mathrm{JJ,} \mathrm{Xu} \mathrm{B,} \mathrm{et} \mathrm{al.} \mathrm{Increased} \mathrm{plasma} \mathrm{C-reactive} \mathrm{protein} \mathrm{level}$ predicts rapid progression of non-target atherosclerotic lesions in patients with stable angina after stenting. Chin Med J (Engl). 2011;124(19):3022-3029.

18. Yun $\mathrm{KH}$, Jeong $\mathrm{MH}$, Oh $\mathrm{SK}$, et al. Response of high-sensitivity C-reactive protein to percutaneous coronary intervention in patients with acute coronary syndrome. Heart Vessels. 2009;24(3):175-180.

19. Holvoet P, de Keyzer D, Jacobs DR. Oxidized LDL and the metabolic syndrome. Future Lipidol. 2008;3(6):637-649.

20. Fiorentino TV, Prioletta A, Zuo P, Folli F. Hyperglycemia-induced oxidative stress and its role in diabetes mellitus related cardiovascular diseases. Curr Pharm Des. 2013;19(32):5695-5703.

21. Lu H, Yao K, Huang D, et al. High glucose induces upregulation of scavenger receptors and promotes maturation of dendritic cells. Cardiovasc Diabetol. 2013;12:80.

22. Nergiz-Unal R, Rademakers T, Cosemans JM, Heemskerk JW. CD36 as a multiple-ligand signaling receptor in atherothrombosis. Cardiovasc Hematol Agents Med Chem. 2011;9(1):42-55.

23. Siegel-Axel D, Daub K, Seizer P, Lindemann S, Gawaz M. Platelet lipoprotein interplay: trigger of foam cell formation and driver of atherosclerosis. Cardiovasc Res. 2008;78(1):8-17.

24. Badrnya S, Schrottmaier WC, Kral JB, et al. Platelets mediate oxidized low-density lipoprotein-induced monocyte extravasation and foam cell formation. Arterioscler Thromb Vasc Biol. 2014;34(3):571-580.

25. Harb D, Bujold K, Febbraio M, Sirois MG, Ong H, Marleau S. The role of the scavenger receptor $\mathrm{CD} 36$ in regulating mononuclear phagocyte trafficking to atherosclerotic lesions and vascular inflammation. Cardiovasc Res. 2009;83(1):42-51.

26. Choromańska B, Myśliwiec P, Choromańska K, Dadan J, Chabowski A. The role of CD36 receptor in the pathogenesis of atherosclerosis. Adv Clin Exp Med. 2017;26(4):717-722.
Journal of Inflammation Research

\section{Publish your work in this journal}

The Journal of Inflammation Research is an international, peer-reviewed open access journal that welcomes laboratory and clinical findings on the molecular basis, cell biology and pharmacology of inflammation including original research, reviews, symposium reports, hypothesis formation and commentaries on: acute/chronic inflammation; mediators of
Dovepress

inflammation; cellular processes; molecular mechanisms; pharmacology and novel anti-inflammatory drugs; clinical conditions involving inflammation. The manuscript management system is completely online and includes a very quick and fair peer-review system. Visit http://www.dove press.com/testimonials.php to read real quotes from published authors. 